\title{
Integrating graphical and natural language specifications to support analysis and testing
}

\author{
Christopher L. Robinson-Mallett \\ Mobility Division, Siemens AG, Berlin \\ c.robinson-mallett@siemens.com
}

\author{
Robert M. Hierons \\ Brunel University London, \\ Uxbridge, United Kingdom \\ rob.hierons@brunel.ac.uk
}

\begin{abstract}
The ongoing trend towards distributed development activities causes a growing need for specification activities and techniques. Each component leads to a large number of specification documents being exchanged, change managed and committed. The quality of the specifications influences the timing, costs and success of the development task. However, the quality of such specifications is often far from optimal, exhibiting gaps, inconsistencies, redundancies, and unbalanced structures. At every release or delivery milestone, acceptance and integration testing take place. Therefore, test-cases have to be created from the requirements exchanged. This paper presents a model-based approach for improving the quality of comprehensive requirements sets. The presented solution is based on a combination of a graphical notation and natural language and can be used to drive model-based testing. The approach has been implemented using state-of-the-art tools. We present experience from field application in the automotive industry.
\end{abstract}

\section{INTRODUCTION}

The ongoing trend towards distributed development activities amongst world-wide teams and the integration of the respective results causes a growing need for specification activities and techniques. Each component leads to a large number of specifications being exchanged, change managed as well as integration and tests committed between the leading and the supplying development teams.

While distributed development projects are not the topic of this paper, our experience with them motivated the work described. The quality of specifications has a significant impact on the efficiency and risks of distributed development activities [1] such as the quality of development results, change management, coordination activities, and testing. Furthermore, the development of dependable software demands traceable processes and provably complete and correct results.

This paper aims at reducing risks of distributed development activities, presenting a method to create and maintain product specifications that are precise, formally structured, and that product engineers find equally easy to understand and to use.

Despite their potential benefits, formal languages (FL) are not widely used because the required expertise is often not present. Instead, natural language (NL) in combination with graphical notations (GN) is a familiar choice for the engineer, with the use of GN helping reduce the size of the NL specification and providing the potential to automate analysis, transformation and testing. An additional benefit is that often there is domain specific technical NL and GN that is common to all groups. Thus a combination of $\mathrm{NL}$ and $\mathrm{GN}$ is practically the de facto standard specification language in distributed development scenarios, although significant drawbacks exist:

1) NL can leave much room for interpretation;

2) NL is local, e.g. a specification written in German is only useful for German speaking groups (this can also affect models);

3) It is difficult to maintain NL and keep it precise, consistent and free of redundancies;

4) Automated processing of NL is difficult;

5) Over time and changes, NL and embedded or referred GN tend to diverge in meaning;

6) GN alone may contribute precise, but only partial information, which demand NL requirements to tell the reader how to interpret it.

Our experience in industrial projects indicates that structure has a significant impact on requirements quality. An unclear structure may contribute to redundancy and so to overly long specifications. Additionally, inconsistencies may be created, if additional requirements deviate from those already in the set.

In the context of this paper a specification is a set of statements (requirements) describing aspects of the product under development. Such statements may possess a type, for instance to denote priority or use of certain information, e.g. differentiate requirements from heading or secondary information. A specification targets a stage of the development process, e.g. product definition, analysis, design, implementation, testing, integration, delivery. Furthermore, specifications may have a certain scope, describing the product as a whole or only parts.

The proposed approach aims to create a model-integrated specification that closes the gap between NL and GN through formalising the structure of the GN specification, while preserving the benefits of NL. We have found that a major benefit is that the resulting specifications provide almost optimal cohesion. In addition, it is possible to apply formal analysis and test generation techniques to the resultant specifications. This paper extends previous work [6] by providing additional information about the formalisation used and by describing a semi-automatic approach to test generation along with associated coverage criteria.

This paper is structured as follows. Section II gives the running example and Section III describes the proposed approach. Section IV explains how the resultant combination of GN and NL can drive testing. Section V describes our experiences of using the proposed approach and associated tool. Section VI 


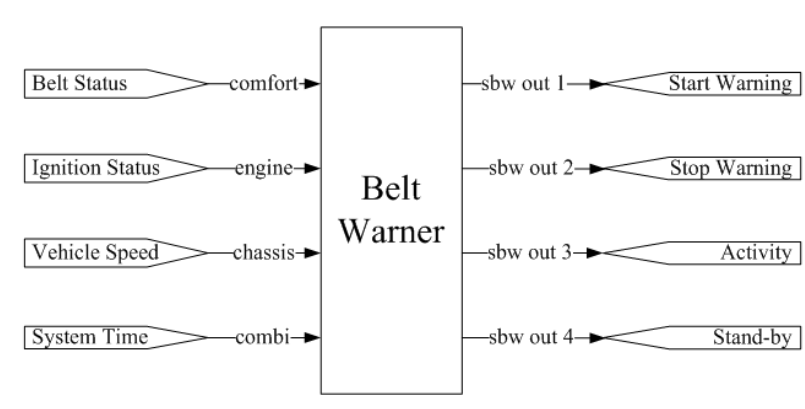

Fig. 1. Block diagram belt-warner example

then describes related work and Section VII summarises the work.

\section{BELT-WARNER EXAMPLE}

The belt-warner example is a simplified extract of the specification of a real driver assistance function that warns the driver at critical speeds that the safety belt is not tightened. The architecture of the system is described using a subset of Block Diagrams [2] [3]. The system behaviour is specified using a sub-set of statecharts [4].

\section{A. Architecture of the belt-warner example}

In the belt-warner example a block diagram specifies the system architecture. Block diagrams are graphical structures consisting of hierarchical blocks, signal flows, signal entry points and signal exit points. A block, represented as a rectangle, may contain a behaviour. Hierarchical blocks may possess architecture and behaviour. Signal flows, represented as edges in the block diagram, specify message routing between blocks. Signal sources and sinks, represented as wedged rectangles, are special blocks used to define system interfaces.

The example architecture of a simple belt-warner in Figure 1 contains a single block, 4 signal sources and 4 signal sinks. The sources define the input interface, which consists of signals Belt Status, Ignition Status, Vehicle Speed, and System Time. The sinks define the output interface, which consists of signals Start Warning, Stop Warning, Activity, and Stand-by.

\section{B. Behaviour of the belt-warner example}

Statecharts is a graphical notation for extended, hierarchical finite state-machines that is applicable to the specification of state-based system behaviour. In the context of the presented approach, a sub-set of statecharts has proved to be useful and will be explained in this section. A complete and detailed description of statecharts is provided in [4].

The nodes of a statechart represent a finite set of system states, while the edges represent transitions between these states. A statechart may process a finite set of inputs and produces a finite set of outputs. A statechart may be extended with data of arbitrary number and types.

A state may contain a finite set of sub-states, one being the initial state, i.e. when changing into the state the initial substate is entered. A transition into a hierarchical state (a state containing sub-states) ends either implicitly in the initial or

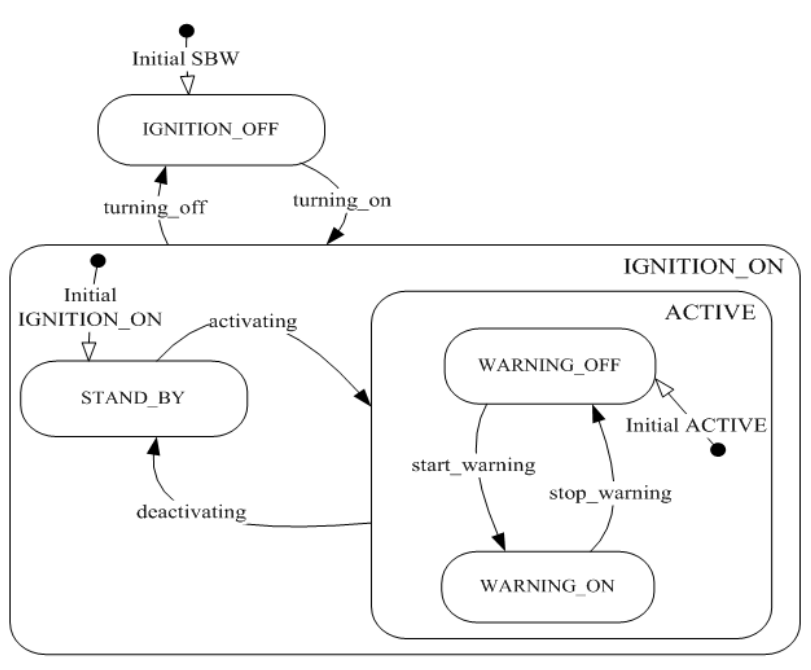

Fig. 2. Statechart for the belt-warner

explicitly in one of the sub-states. An atomic state does not contain sub-states. A state-invariant specifies conditions that must hold when the system is in the corresponding state.

A state transition has an initial and a final state. A group transition starts from a hierarchical state $S_{h}$; it can be triggered from any sub-state of $S_{h}$. The trigger of transition $t$ specifies conditions on inputs and data under which $t$ executes. Furthermore, in a transition it is possible to specify data operations and outputs. We use predicate logic to specify the above.

The statechart in Figure 2 contains 4 atomic states, 2 hierarchical states and 9 transitions, of which 3 are initial transitions and 4 are group transitions. It describes the system behaviour in use, when turning it on/of, activating, deactivating the function and situations in which the belt warning is started or stopped.

\section{Model-InTEgRated ReQuirements SPECIFICATION}

As previously discussed, the proposed approach is based on integrating GN and NL, which is achieved by mapping each 'basic model element' of the graphical model to a corresponding textual section (called a chapter).

This process is applicable to a variety of modeling notations, if these can be mapped into finite structures, such as graphs. Given an NL specification of a system (for the ease of explanation initially without GN parts), we create a modelintegrated specification as follows.

1) Break down the specification into atomic statements.

2) Give each atomic statement a type of either information or requirement.

3) Create a GN of the system's high-level architecture.

4) Create a NL chapter structure based on the architecture:

- each node in the GN relates to a chapter,

- each edge in the GN relates to a chapter.

5) Relocate atomic statements into the chapter structure.

6) Analyse residual atomic statements and either discard or redesign GN and go back to 3 . 


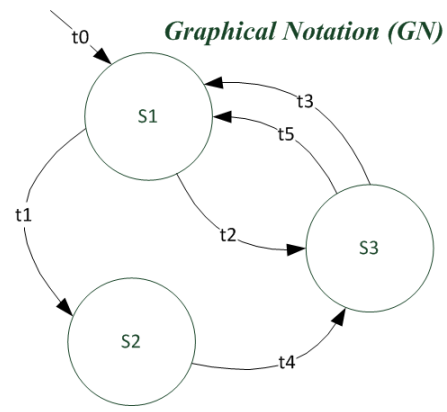

\begin{tabular}{|l} 
Specification Structure (NL) \\
Chapter 1 States \\
Chapter 1.1 State S1 \\
Chapter 1.2 State S2 \\
Chapter 1.3 State S3 \\
Chapter 2 Transitions \\
Chapter 2.1 Transition t0 \\
Chapter 2.2 Transition $t 1$ \\
Chapter 2.3 Transition $t 2$ \\
Chapter 2.4 Transition $t 3$ \\
Chapter 2.5 Transition $t 4$ \\
Chapter 2.6 Transition $t 5$
\end{tabular}

Fig. 3. Example model-integrated finite state machine

7) Review each chapter and eliminate incompleteness, inconsistencies and redundancies.

8) Optionally, complement NL specification with conditional statements defining feasible paths and further system properties, e.g. state invariants.

This results in a semi-formal specification in the form of a chapter structure; specification statements remain in NL. For instance, if the GN is a finite state machine, then the model elements are states and transitions, and a chapter is created for each of these. Figure 3 presents the example of an FSM and the NL structure generated.

In this paper we outline how GN in the form of labelled directed graphs (LDGs) can be used. The GN part may have a specific (separate) semantics associated with the language originally used. We need to retain this information to correctly interpret different languages such as statecharts and block diagrams. We have developed a set of semantics for automotive controller functions but anticipate that different semantics might be used in other domains. In practice, we have found LDGs to cover a large and useful variety of modelling notations. However, there might be notations, e.g. sequence diagrams and use-case diagrams, where there is no natural mapping to LDGs. In such cases we suggest that different GN-notations are used, e.g. multi-graphs.

In practical software and systems development, the variety of GN used often depends on the domain (e.g. statecharts, activity diagrams, Petri nets, block diagrams, or fault trees). To make the model-integration approach applicable to a maximum number of GNs, it splits a GN into an abstract graphical structure, whose elements will be given semantics via NL, and a semantics that comes from the original graphical modelling language used. For the example in Figure 3, the semantics includes the information that the graph represents a finite state machine: nodes represent states; edges represent transitions. The graph of a GN thus represents the topology. Mapping a GN to abstract graphs provides the benefit that we can have a common approach and toolset for a range of GNs.

Conditional statements form an important part of many modelling notations. For example, statecharts has state invariants and trigger conditions on edges. In the presented approach, predicate logic may be used to specify conditional structures in the NL part of the model-integrated specification; we will see that this facilitates semi-automated test generation. It is possible to structure this NL to form logical trees, where leaves are atomic logical statements in NL and the other nodes represent the logical operators OR, AND and XOR.

\section{A. An abstract model}

A labelled directed graph $G$ is defined by a tuple $\left(L, V, V_{0}, E, f_{V}\right)$ in which $L$ is the set of labels; $V$ is the finite set of nodes; $V_{0} \subset V$ is the set of initial nodes; $E \subseteq V \times L \times V$ is the finite set of edges (edge $\left(v, l, v^{\prime}\right)$ has source node $v$, destination node $v^{\prime}$ and label $l$ ); and $f_{V}: V \rightarrow L$ is the node label function that maps each node to a corresponding label.

The nodes and edges of graph $G$ provide the structure of the model. The labels allow us to add semantics to these.

$L$ can be any suitably large set since the individual labels can be separately mapped to the required information (such as invariants). In practice, we have found it simplest to use strings, with a label denoting, for example, the name of a state or a function. Information regarding the semantics associated with a label is supplied in a separate table. Tables I and II give such information for the belt-warner system.

TABLE I

LDG OF BELT-WARNER BLOCK DIAGRAM

$$
\begin{aligned}
& \begin{aligned}
G_{b d} & =\left(\quad\left(n_{0}, \text { Belt Status, source }\right),\right. \\
V & =\{\quad \text {, }
\end{aligned} \\
& \text { ( } n_{1}, \text { Ignition Status, source), } \\
& \left(n_{2},\right. \text { Vehicle Speed, source), } \\
& \text { ( } n_{3}, \text { System Time, source), } \\
& \left(n_{4}, \text { Simple Belt Warner, node }\right) \text {, } \\
& \left(n_{5}, \text { Start Warning, sink }\right) \text {, } \\
& \left(n_{6},\right. \text { Stop Warning, sink), } \\
& \left(n_{7}, \text { Belt Warner Activity, sink }\right) \text {, } \\
& \left.\left(n_{8}, \text { Belt Warner Stand -By, sink }\right)\right\} \text {, } \\
& E=\left\{\quad\left(\left(n_{0}, n_{4}\right), \text { comfort }\right),\right. \\
& \left(\left(n_{1}, n_{4}\right), \text { engine }\right), \\
& \left(\left(n_{2}, n_{4}\right), \text { chassis }\right) \text {, } \\
& \left(\left(n_{3}, n_{4}\right), c o m b i\right) \text {, } \\
& \left(\left(n_{4}, n_{5}\right), \text { sbwout } 1\right) \text {, } \\
& \left(\left(n_{4}, n_{6}\right), \text { sbwout } 2\right) \text {, } \\
& \left(\left(n_{4}, n_{7}\right), \text { sbwout } 3\right) \text {, } \\
& \left.\left.\left(\left(n 4, n_{8}\right), \text { sbwout } 4\right)\right\}\right)
\end{aligned}
$$

TABLE II

LDG OF BELT-WARNER STATECHART

$$
\begin{aligned}
& G_{s c}=( \\
& V=\left\{\quad\left(n_{0}, \text { IGNITION_OFF, node }\right),\right. \\
& \left(n_{1},\right. \text { IGNITION_ON, hierarchical node), } \\
& \left(n_{2}, S T A N D \_B \bar{Y}, \text { node }\right) \text {, } \\
& \text { ( } n_{3}, A C T I V E \text {, hierarchical node), } \\
& \left(n_{4}, W A R N I N G \_O F F, \text { node }\right) \text {, } \\
& E=\left\{\begin{array}{l}
\left(n_{5}, W\right. \text { ARNING_ON, } \\
\left(\left(-, n_{0}\right), \text { Initial } S B W\right),
\end{array}\right. \\
& \left(\left(n_{0}, n_{1}\right) \text {, turning_on }\right) \text {, } \\
& \left(\left(n_{1}, n_{0}\right) \text {, turning_off }\right) \text {, } \\
& \left(\left(-, n_{2}\right) \text {, Initial IGNITION_ON }\right) \text {, } \\
& \left(\left(n_{2}, n_{3}\right), \text { activating }\right) \text {, } \\
& \left(\left(-, n_{4}\right),\right. \text { Initial ACTIVE), } \\
& \left(\left(n_{4}, n_{5}\right), \text { start_warning }\right) \text {, } \\
& \left.\left.\left(\left(n_{5}, n_{4}\right), \text { stop_warning }\right)\right\}\right)
\end{aligned}
$$




\section{B. Semantics}

The process of mapping a graphical model into LDGs can lead to some loss of information that corresponds to elements of the semantics of the GN used. For example, if we map a statechart into an LDG then the resultant graph does not contain information such as the fact that a node represents a state and an edge represents a transition from one state to another. This additional semantic information must be retained; in the proposed approach the identity of the original GN is retained so that it is possible to apply analysis techniques that depend on this semantics. Furthermore, it is possible to attach specific properties $p_{0}, \ldots, p_{n}$ for each model element type. For instance, properties may be used to define a specific chapter structure for each model element type, e.g. a state chapter must contain an invariant definition and a transition chapter must contain a trigger definition.

Tuples are used to define the semantics associated with elements of the LDG; a tuple relates a graph element to a GN element type and additional information, e.g. a template for the chapter title. Chapter title templates are string expression, possibly with placeholders, e.g. for a system name or a model element name. An example of a modeling notation semantics for block diagrams is presented in Table III. The graph of the modeling notation consists of the modeling elements hierarchical node, node, edge, source, and sink. Each modeling element is mapped to the appropriate element of block diagrams, e.g. a node is a block, an edge is a signal flow.

TABLE III SEMANTICS OF BLOCK DIAGRAM

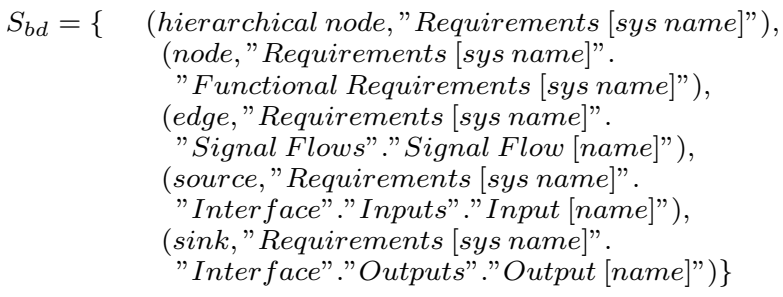

An example of a modeling notation semantics for state charts is presented in Table IV. The graph of the modeling notation consists of the modeling elements hierarchical node, node, and edge. Compared to block diagrams there is no need to consider sinks and sources. Each of these modeling elements is mapped to the appropriate element of statecharts, e.g. a node is a state, an edge is an transition.

TABLE IV

SEMANTICS OF STATECHART

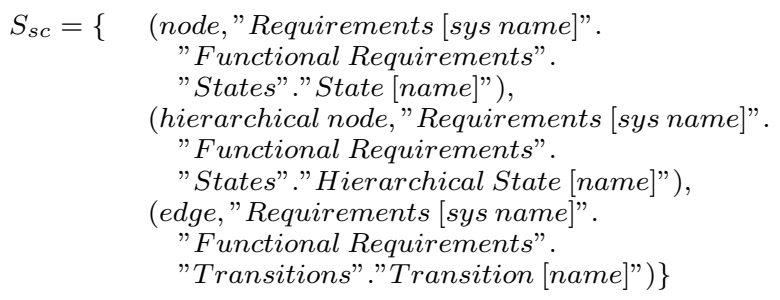

\section{Generating Specification Structures}

A specification structure consists of hierarchically structured chapters, where each chapter provides semantics for an element of the GN. The title of a chapter is created from information regarding a graph element and its semantics properties. Identifiers label graph elements; these provide a one-to-one mapping between chapters and graph elements. For example, it is possible to create a specification structure for the belt-warner through two steps: generate chapters from the Block Diagram (bold chapters); generate chapters from the Statechart. Figure 4 gives the resulting structure.

\begin{tabular}{|l|}
\hline Chapter X Requirements Belt Warner \\
Chapter X.1 Interface \\
Chapter X.1.1 Inputs \\
Chapter X.1.1.1 Input Belt Status \\
Chapter X.1.1.2 Input Ignition Status \\
Chapter X.1.1.3 Input Vehicle Speed \\
Chapter X.1.1.4 Input System State \\
Chapter X.1.2 Outputs \\
Chapter X.1.2.1 Output Start Warning \\
Chapter X.1.2.2 Output Stop Warning \\
Chapter X.1.2.3 Output Activity \\
Chapter X.1.2.4 Output Stand-By \\
Chapter X.2 Functional Requirements \\
Chapter X.2.1 States \\
Chapter X.2.1.1 Hierarchical State IGNITION_ON \\
Chapter X.2.1.2 Hierarchical State ACTIVE \\
Chapter X.2.1.3 State IGNITION_OFF \\
Chapter X.2.1.4 State STAND_BY \\
Chapter X.2.1.5 State NOT_WARNING \\
Chapter X.2.1.6 State WARNING \\
Chapter X.2.2 Transitions \\
Chapter X.2.2.1 Transition Initial_SBW \\
Chapter X.2.2.2 Transition Initial_IGNITION_ON \\
Chapter X.2.2.3 Transition Initial_ACTIVE \\
Chapter X.2.2.4 Transition turning_on \\
Chapter X.2.2.5 Transition turning_off \\
Chapter X.2.2.6 Transition activating \\
Chapter X.2.2.7 Transition deactivating \\
Chapter X.2.2.8 Transition start_warning \\
Chapter X.2.2.9 Transition stop_warning \\
\hline
\end{tabular}

Fig. 4. Model-integrated structure of belt-warner specification

\section{Specification Chapters}

A specification chapter has a title and a set of specification entries. Each specification entry can have a specific type, e.g. requirement, information, data type, physical constraint.

Each GN element type can have a specific specification chapter structure, e.g. a state chapter may contain an invariant while a transition chapter may contain initial state, final state, and trigger. As a result, for each modelling notation we use specification chapter templates that define a hierarchical structure of specification entries for each model element type. Common attributes for specification entries are types, owner, date of creation and last edit, or a history record. For modelintegration a specification type attribute is defined (Table V).

Logical expressions may be used to define conditions that complement GN, e.g. triggers and invariants. These conditions 
TABLE V

SPECIFICATION OBJECT TyPES

\begin{tabular}{|l|l|}
\hline Type & Description \\
\hline heading & a structure element \\
\hline information & explanations and comments \\
\hline requirement & the specification of a single required product property \\
\hline
\end{tabular}

may be specified using NL. Naturally, complex conditions can be split into atomic expressions, these being structured into a tree. An example from the belt-warner is given in Table VI.

TABLE VI

CONDITION SPECIFICATION TYPES

\begin{tabular}{|l|l|}
\hline Type & description \\
\hline condition & atomic condition \\
\hline XOR & exclusive disjunctive composition of conditions \\
\hline OR & disjunctive composition of conditions \\
\hline AND & conjunctive composition of conditions \\
\hline
\end{tabular}

Since the atomic conditional expressions remain in NL, the graph traversal produces concatenated NL expressions, which only a human reader may check for validity. However, the ability to automatically generate traces from conditional GN was found to be very beneficial.

Returning to the running example, Table VII presents a specification chapter template for states ('tbd' denotes information to be added by the user). Likewise, a chapter template for transitions is presented in Table VIII.

TABLE VII

Specification Chapter Template Belt-Warner State

\begin{tabular}{|l|l|l|}
\hline Type & Short & Text \\
\hline heading & - & Chapter X.2.1.3 State IGNITION_OFF \\
\hline information & Context & tbd \\
\hline AND & Invariant & tbd \\
\hline
\end{tabular}

TABLE VIII

SPECification Chapter TEMPLATE BELT-WARNER TRANSition

\begin{tabular}{|l|l|l|}
\hline Type & Short & Text \\
\hline heading & - & Chapter X.2.2.4 Transition turning_on \\
\hline information & Context & tbd \\
\hline requirement & Initial State & tbd \\
\hline requirement & Final State & tbd \\
\hline AND & Trigger & tbd \\
\hline
\end{tabular}

Finally, the specification chapters are complemented manually with details in NL. As an example for the use of specification types, Table IX presents requirements for the activation hysteresis of the simple belt-warner.

Logical conditions like the activation hysteresis are often found in specification documents. The model-integrated specification can be extended with formalised conditional specifications resulting in an extended model-integrated specification; graph operations are still applicable. Again, the formalisation applies only to the structure of the conditional specifications, the conditional statements remain NL.
TABLE IX

BELT-WARnER HySTERESIS SPECIFICATION

\begin{tabular}{|l|l|}
\hline Type & Text \\
\hline information & $\begin{array}{l}\text { The driver must not be distracted through } \\
\text { unnecessary belt-warnings at low speeds. }\end{array}$ \\
\hline requirement & $\begin{array}{l}\text { The belt-warner shall be activated, } \\
\text { if vehicle speed exceeds } 15 \mathrm{mph} .\end{array}$ \\
\hline requirement & $\begin{array}{l}\text { The belt-warner shall be deactivated, } \\
\text { if vehicle speed drops below } 12 \mathrm{mph} .\end{array}$ \\
\hline requirement & $\begin{array}{l}\text { The belt-warner shall be deactivated, } \\
\text { if a relevant malfunction occurs. }\end{array}$ \\
\hline
\end{tabular}

TABLE $X$

BELT-WARNER IGNITION OFF INVARIANT SPECIFICATION

\begin{tabular}{|l|l|}
\hline Type & Text $(\bullet-$ level $1, \circ-$ level $2, \star-$ level 3$)$ \\
\hline AND & System resides in IGNITION_OFF, if \\
\hline condition & $\bullet$ Belt-warner ECU not powered. \\
\hline
\end{tabular}

TABLE XI

BELT-WARNER WARNING OFF INVARIANT SPECIFICATION

\begin{tabular}{|l|l|}
\hline Type & Text $(\bullet-$ level $1, \circ-$ level $2, \star-$ level 3$)$ \\
\hline AND & System resides in $W A R N I N G \_O F F$, if \\
\hline condition & $\bullet$ No warning sound emitted. \\
\hline condition & $\bullet$ Seat-belts fastened. \\
\hline
\end{tabular}

Examples of specifications of state invariants of the beltwarner are presented in Tables X and XI; others are similar.

Examples of specifications of the triggering conditions of the belt-warner example are presented in Tables XII and XIII.

TABLE XII

BELT-WARNER INITIAL CONDITION SPECIFICATION

\begin{tabular}{|l|l|}
\hline Type & Text $(\bullet-$ level $1, \circ-$ level $2, \star-$ level 3$)$ \\
\hline AND & Seat-belt warner turns on, if \\
\hline condition & $\bullet$ System precondition is TRUE. \\
\hline
\end{tabular}

TABLE XIII

BELT-WARNER START WARNING CONDITION SPECIFICATION

\begin{tabular}{|l|c|}
\hline Type & Text $(\bullet-$ level $1, \circ-$ level $2, \star-$ level 3$)$ \\
\hline XOR & Seat-belt warning must start, if \\
\hline condition & $\bullet$ The driver seat-belt is unfastened. \\
\hline AND & $\bullet$ passenger seat \\
\hline condition & $\circ$ Passenger seat is occupied. \\
\hline condition & $\star$ Passenger seat-belt is unfastened. \\
\hline
\end{tabular}

The conditional specifications complement the belt-warner model in Figure 2, resulting in a non-deterministic modelintegrated specification. This model-integrated specification can be input to further automate analysis and testing steps.

\section{Model-Based Test-Case Specification}

In this section, we present an approach to apply modelbased test-case generation to model-integrated specifications.

\section{A. Creating Test-Cases}

The presented test-case creation approach combines automated test-case generation with experienced based testing. We 


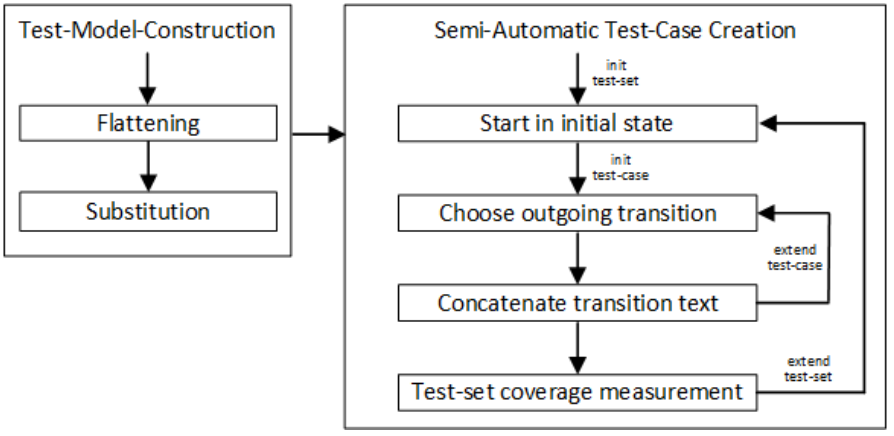

Fig. 5. Model-based testing process

outline this for statecharts. Initially, the statechart is flattened and some modeling elements are substituted. Conditional trigger specifications are transformed into disjunctive normalform; for each conjunct a corresponding transition is added to the model. The resulting model is a graph to which one can apply common traversal and coverage analysis algorithms.

We have found that it is not always sensible to directly apply graph-traversal algorithms since the GN might not capture all of the relevant information. For example, a specification might contain a cycle that must be repeated a given number of times before it can be left; we could encode this information in the graph by unfolding the cycle but this might lead to a significant increase in the size of the graph. In addition, the GN might not capture important domain knowledge and may contain non-determinism that results from abstraction. Nevertheless, automation remains a key technology to efficiently handle large specifications and reduce the potential for error in carrying out routine tasks. As a result, we have developed an interactive test case generation process that allows the user's domain knowledge to be utilised. After each step, the tool reports the coverage achieved to allow the user to reason about test-case quality. Based on this, the user either decides to terminate or chooses the next transition to take.

The testing process presented in Figure 5 is divided in two phases: automated test-model construction, i.e. flattening and substitution, and semi-automatic test-case generation. A software tool uses flattening and substitution operations to generate a testing model from the model-integrated specification.

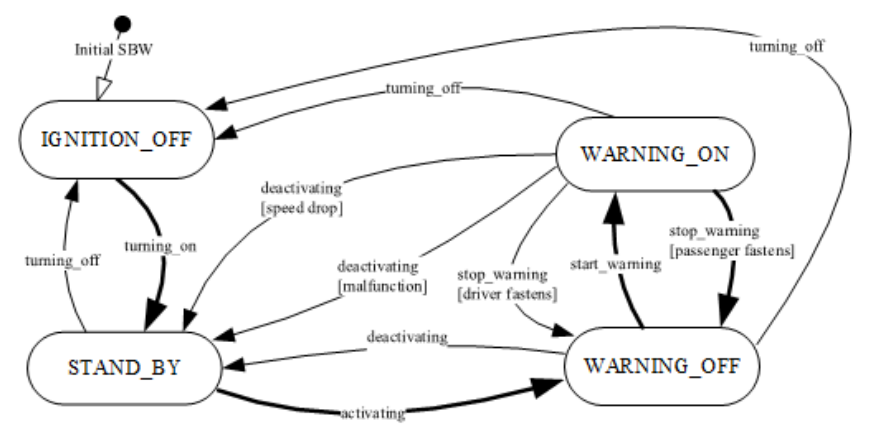

Fig. 6. Test-Model Belt-Warner

The resulting testing model for the belt-warner example is
TABLE XIV

TEST-CASE EXAMPLE

\begin{tabular}{|l|l|l|}
\hline No. & Input & Output \\
\hline 0 & [Initial SBW] & [Invariant IGNITION_OFF] \\
& System precondition is TRUE. & Belt-warner ECU not powered. \\
\hline 1 & [Trigger turning_on] & [Invariant IGNITION_ON] \\
& Ignition key turned ON. & Belt-warner ECU powered. \\
& Error memory is empty. & [Invariant STAND_BY] \\
& [Trigger Initial IGNITION_ON] & Belt-warner signals stand-by. \\
& Set power status bit $=1$. & \\
\hline 2 & [Trigger activating] & [Invariant IGNITION_ON] \\
& Vehicle speed $\geq 25 \mathrm{~km} / \mathrm{h}$. & Belt-warner ECU powered. \\
& [Trigger Initial ACTIVE] & Error memory is void. \\
& Set activity bit $=1$. & [Invariant ACTIVE] \\
& & Belt warner signals activity. \\
& & Vehicle speed $>25 \mathrm{~km} / \mathrm{h}$. \\
& & [Invariant WARNING_OFF] \\
& & No warning sound emitted. \\
& & Seat belts fastened. \\
\hline 3 & [Trigger start_warning] & [Invariant IGNITION_ON] \\
& Passenger seat occupied. & Belt-warner ECU powered. \\
& Passenger seat-belt unfastened. & Error memory is void. \\
& & [Invariant ACTIVE] \\
& & Belt warner signals activity. \\
& & Vehicle speed $>25 \mathrm{~km} / \mathrm{h}$. \\
& & [Invariant WARNING_ON] \\
& & A warning sound emitted. \\
& & Passenger seat-belt unfastened. \\
\hline 4 & [Trigger stop_warning] & [Invariant IGNITION_ON] \\
& Warning emitted. & Belt-warner ECU powered. \\
& Driver seat-belt fastened. & Error memory is void. \\
& Passenger seat occupied. & [Invariant ACTIVE] \\
& Passenger seat-belt fastened. & Belt warner signals activity. \\
& & Vehicle speed $>25 \mathrm{~km} / \mathrm{h}$. \\
& & [Invariant WARNING_OFF] \\
& & No warning sound emitted. \\
& & Seat-belts fastened. \\
\hline & & \\
& &
\end{tabular}

presented in Figure 6. The testing model is used by another software tool to support the test-case specification process by advising the test engineer. Test-case creation starts with an empty sequence in the initial state (the initial transition may add text to the test-case too, but for now we will ignore this). The software tool supports the test-engineer by offering the current state's outgoing transitions and its textual contents, which may be chosen to extend the test-case. When the testengineer selects a transition its textual content is appended to the test-case and the current state is changed.

Once a test-case is finished, it is added to a test-set (a collection of test-cases). The tool applies coverage metrics to a test-set so that the test engineer can decide whether further test-cases should be added. Table XIV gives a test-case created using the tool from the belt-warner model. The test-case covers the edges marked bold in the model presented in Figure 6.

\section{B. Test coverage criteria}

Let us suppose that $S$ is a model-integrated specification, $G_{S}$ is an $L D G$ generated from $S$, and $G_{T}$ is a test-model derived from $S$. Further, suppose that $t_{S}$ is a test suite. We have several possible coverage metrics.

Edge coverage $C_{G}$ is the fraction of edges of $G_{S}$ that are covered when executing $t_{S}$. Condition coverage $C_{S}$ is the fraction of conditional statements (within structured logical 
expressions) of $S$ covered when executing $t_{S}$. Trigger coverage $C_{T}$, is the fraction of edges of the test-model $T$ that are covered when executing $t_{S}$. For effort estimation, an upper bound on the number of edges in $T$ is provided by taking the sum, over the edges of $G_{S}$, of the number of conditions in the DNF form of the triggers. The test-case presented in Table XIV results in the coverage rates presented in Table XV.

TABLE XV

BELT-WARNER TEST COVERAGE EXAMPLE

\begin{tabular}{|l|l|l|l|}
\hline Transition & $C_{G}$ & $C_{S}$ & $C_{T}$ \\
\hline Initial ACTIVE & $100 \%$ & $100 \%$ & $100 \%$ \\
\hline Initial IGNITION_ON & $100 \%$ & $100 \%$ & $100 \%$ \\
\hline Initial SBW & $100 \%$ & $100 \%$ & $100 \%$ \\
\hline activating & $100 \%$ & $100 \%$ & $100 \%$ \\
\hline deactivating & $0 \%$ & $0 \%$ & $0 \%$ \\
\hline start_warning & $100 \%$ & $50 \%$ & $50 \%$ \\
\hline stop_warning & $100 \%$ & $50 \%$ & $100 \%$ \\
\hline turning_off & $0 \%$ & $0 \%$ & $0 \%$ \\
\hline turning_on & $100 \%$ & $100 \%$ & $100 \%$ \\
\hline
\end{tabular}

\section{CAse Studies}

The model-integration approach and tool have been used in a number of development projects in industry: system specification of various parking assistance systems using block diagrams and statecharts; functional specification of emergency braking systems using statecharts; system specification of camera-based surround view systems; system, function and test-case specifications of the control of an automated manual gearbox; architecture, function and user interface specifications of a database management tool; and architecture and function of a software-controlled medical device.

Among the specified driver assistance systems are 4 parking systems, 2 brake-assistance systems, and a camera-based traffic information system, for which it was found that a major benefit lies in the clear structure and transparency of the model-integrated specification. The largest specification included 5 functional variants of a parking system containing more than 2000 requirements, which were structured using 8 statechart models, of which the largest contained 40 states and 69 transitions. Frequently it was seen that the specifications would increase in size. Even though redundancies can be eliminated completely, the model-based approach enforces completeness and the additional, missing, requirements caused the specification to grow.

Functional specifications of an automated gear-box were created using the model-integration tool; experience data is presented in Table XVI. Two requirements engineers took four months to create model-integrated specifications from highlevel functional specification. Since the functional specifications were found to be incomplete and inconsistent, workshops were held with the system developers to elicit missing requirements and to remove specification faults. The model-integrated specifications were complemented with testing information, so that the same specifications could be used to generate testcases for manual and automatic execution.
TABLE XVI

Automatic Gearbox ApPlication Statistics

\begin{tabular}{|l|l|l|l|}
\hline Component & Requirements & Nodes & Edges \\
\hline Coasting & 315 & 18 & 36 \\
\hline Creeping & 275 & 15 & 34 \\
\hline Garage Shift & 268 & 12 & 34 \\
\hline Launch & 262 & 16 & 32 \\
\hline Shift Quality & 293 & 14 & 21 \\
\hline Stalling & 144 & 11 & 20 \\
\hline Startup\&Shutdown & 117 & 17 & 26 \\
\hline
\end{tabular}

The model-integration has also been used to specify a medical system consisting of 7 major components. Experience data is presented in Table XVII.

TABLE XVII

Medical Device Application Statistics

\begin{tabular}{|l|l|l|l|}
\hline Component & Requirements & Nodes & Edges \\
\hline ECU & 91 & 6 & 8 \\
\hline Sensors & 98 & 8 & 15 \\
\hline Actuators & 92 & 8 & 14 \\
\hline Radio Arch. & 17 & 2 & 2 \\
\hline Radio Func. & 83 & 5 & 15 \\
\hline Radio Master & 92 & 11 & 15 \\
\hline Radio Client & 71 & 8 & 13 \\
\hline
\end{tabular}

The model-integrated specification approach is in use. To specify graphical user interfaces, a specific graphical notation and semantics have been developed that represents the graphical widgets and their placement on the screen. Functional behaviour, in the form of a statechart, can be attached to widgets. The project, including the specification approach, has been assessed and certified for SPICE level 1 [5].

TABLE XVIII

COMPARISON OF APPLICATION STATISTICS IN DEVELOPMENT PROJECTS

\begin{tabular}{|l|l|l|l|l|l|}
\hline System & Comp. & Models & Nodes & Edges & Effort[h] \\
\hline Parking 1 & 4 & 8 & 40 & 69 & 500 \\
\hline Parking 2 & 4 & 5 & 19 & 38 & 650 \\
\hline Brake 1 & 1 & 2 & 6 & 8 & 700 \\
\hline Brake 2 & 1 & 2 & 15 & 18 & 800 \\
\hline Gearbox & 7 & 7 & 103 & 203 & 1100 \\
\hline SW Tool & 3 & 26 & 133 & 235 & 900 \\
\hline Medical Device & 4 & 7 & 48 & 82 & 400 \\
\hline
\end{tabular}

Table XVIII presents statistics regarding specifications created using the approach presented. The rows refer to the specified systems. The columns contain counts of components, models, states and transitions included in the specifications.

Table XVIII shows very different efficiencies (efforts per node, edge, model, or component), where effort is a rough approximation of the number of person hours required to create and maintain the specification during the project. The parking and brake assist system projects were the first applications of the approach and were involved in the development of the method and tool. Thus the comparably low efficiency is caused by less automation and a less mature approach. Furthermore, we found that the number of components and models are individual decisions made during the early phases of each 
project. The influence of such decisions on the efficiency of development activities is hard to determine. However, as a rule of thumb, we found during the further projects that specifying and maintaining a chapter takes approximately up to two days.

\section{RELATED WORK}

The basic approach described in this paper has previously been presented [6]. This paper provides additional details and formalisation as well as an extended example and experience data. An additional extension is the inclusion of a semiautomatic model-based testing approach and associated coverage criteria. The model-integration approach contributes to the $\mathrm{RE}$ reference model presented in [7] as it provides a method to systematically integrate and relate artefacts (as domain knowledge) into artefacts of requirements and specifications.

The presented approach contributes to the concept of viewpoints resolution presented in [8] through abstraction and unification of document structures that significantly eases the comparison and gap analysis of different viewpoints. The extension of the presented approach to methods for the identification of missing and wrong facts over a set of comprehensive model-integrated specifications is a topic of ongoing work and inspired by the viewpoints analysis presented in [8].

In [6] we noted that structure has a significant impact on the quality of requirements sets. Previous work has used clustering to automatically restructure comprehensive requirements sets [9]. In contrast, model-integration takes advantage of the knowledge and experience of the human reader, who is supported by an improved document structure. Similar approaches have been presented on the integration of textual and graphical modeling languages, e.g. [10], [11], although these do not consider natural language as a textual language.

There are many approaches to model-based testing. However, they typically require the existence of a model in a formal language such as a finite state machine [12] or labelled transition system [13], possibly enriched by aspects such as time [14] or probabilities [15], [16]. While NL explanations can complement a model in such a language, the NL is not normally integrated with the model.

\section{CONCLUSIONS}

The model-integrated approach has been presented, with this making it possible to seemlessly integrate textual specifications and models, resulting in a semi-formal specification. The level of formalisation of the resulting specifications makes it possible to use formal analyses and graph operations, e.g. testcase generation. The benefits include the following:

1) formal definition of requirements completeness;

2) efficiency through automation;

3) fewer manual faults through automation;

4) improved clarity through use of the model as a document structure;

5) fewer redundancies and inconsistencies through formal soundness criteria and checking capabilities;

6) no divergence of the GN and NL parts of a specification;

7) eased and accelerated maintenance and change.
The approach has been implemented in a tool. It has been applied in several projects in industry over a period of 6 years.

Future work will aim at the reimplementation of the tool independent of specific commercial requirements management systems and that utilises the model-integration approach. Furthermore, an extended approach is under development that allows for bidirectional changing of models, i.e. to transfer changes of the model from NL into GN and vice versa. Currently, it is only possible to change the model through manually changing the GN-part and transferring these changes automatically to the NL-part. There may also be scope to automatically derive some structure from NL.

\section{REFERENCES}

[1] T. Gilb, "No cure no pay: How to contract for software services," Comput. Sci. Inf. Syst., vol. 4, no. 1, pp. 29-41, 2007.

[2] J. W. Nilsson, Electric circuits (4. ed.), ser. Addison-Wesley series in electrical and computer engineering. Addison-Wesley, 1993.

[3] M. K. Stojcev, "John p. hayes, computer architecture and organization, third ed., mcgraw-hill book company, inc., boston, 1988, softcover, pp 604, plus xiv, ISBN 0-07-115997-5," Microelectronics Reliability, vol. 46, no. 1, pp. 196-197, 2006

[4] D. Harel, "Statecharts in the making: a personal account," Commun. $A C M$, vol. 52, no. 3, pp. 67-75, 2009.

[5] I. O. for Standardization, "Software process improvement and capability determination (spice)," Tech. Rep., 2012.

[6] C. Robinson-Mallett, "An approach on integrating models and textual specifications," in Second IEEE International Workshop on ModelDriven Requirements Engineering, MoDRE 2012, Chicago, IL, USA, September 24, 2012, 2012, pp. 92-96.

[7] C. A. Gunter, E. L. Gunter, M. Jackson, and P. Zave, "A reference model for requirements and specifications," IEEE Software, vol. 17, no. 3, pp. 37-43, 2000. [Online]. Available: http://dx.doi.org/10.1109/52.896248

[8] J. C. S. do Prado Leite and P. Freeman, "Requirements validation through viewpoint resolution," IEEE Trans. Software Eng., vol. 17, no. 12, pp. 1253-1269, 1991.

[9] A. Ferrari, S. Gnesi, and G. Tolomei, "Using clustering to improve the structure of natural language requirements documents," in Requirements Engineering: Foundation for Software Quality - 19th International Working Conference, REFSQ 2013, Essen, Germany, April 8-11, 2013. Proceedings, 2013, pp. 34-49.

[10] L. Engelen and M. van den Brand, "Integrating textual and graphical modelling languages," Electr. Notes Theor. Comput. Sci., vol. 253, no. 7, pp. 105-120, 2010.

[11] M. Scheidgen, "Textual modelling embedded into graphical modelling," in Model Driven Architecture - Foundations and Applications, 4th European Conference, ECMDA-FA 2008, Berlin, Germany, June 9-13, 2008. Proceedings, 2008, pp. 153-168.

[12] R. M. Hierons and U. C. Türker, "Parallel algorithms for testing finite state machines: Generating UIO sequences," IEEE Transactions on Software Engineering, vol. 42, no. 11, pp. 1077-1091, 2016.

[13] J. Tretmans, "Model based testing with labelled transition systems," in Formal Methods and Testing, ser. Lecture Notes in Computer Science, vol. 4949. Springer, 2008, pp. 1-38.

[14] C. Gaston, R. M. Hierons, and P. L. Gall, "An implementation relation and test framework for timed distributed systems," in 25th IFIP WG 6.1 International Conference on Testing Software and Systems (ICTSS 2013), ser. Lecture Notes in Computer Science, vol. 8254. Springer, 2013, pp. 82-97.

[15] M. Gerhold and M. Stoelinga, "Model-based testing of probabilistic systems," in Fundamental Approaches to Software Engineering - 19th International Conference, FASE 2016, ser. Lecture Notes in Computer Science, vol. 9633. Springer, 2016, pp. 251-268.

[16] G. H. Walton, J. H. Poore, and C. J. Trammell, "Statistical testing of software based on a usage model," Softw., Pract. Exper., vol. 25, no. 1, pp. $97-108,1995$ 